\title{
Revista Española de
}

Nutrición Humana y Dietética

\section{Spanish Journal of Human Nutrition and Dietetics}

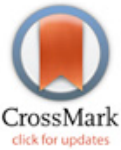

www.renhyd.org

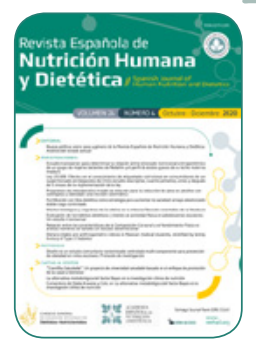

\author{
ARTÍCULO DE INVESTIGACIÓN
}

\section{Fortificación con fibra dietética como estrategia para aumentar la saciedad: ensayo aleatorizado doble ciego controlado}

\author{
José Luis Pino Villalón ${ }^{\mathrm{a}, *}$, Marianela Rojas Muñoz ${ }^{\mathrm{a}}$, Bárbara Orellana Saez ${ }^{\mathrm{a}}$, Jorge Torres Mejías ${ }^{\mathrm{a}}$

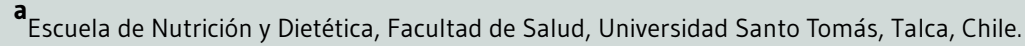 \\ *jpino9@santotomas.cl
}

Editor Asignado: Miguel Ángel Lurueña. Comité Editorial de la Revista Española de Nutrición Humana y Dietética. Pamplona, España.

Recibido el 11 de marzo de 2020; aceptado el 24 de mayo de 2020; publicado el 29 de junio de 2020.

\section{PALABRAS CLAVE}

Fenómenos

Fisiológicos de la

Nutrición;

Regulación del

Apetito;

Apetito;

Saciedad;

Respuesta de

Saciedad;

Escala Visual

Analógica;

Fibras de la Dieta;

Desayuno;

Adulto.

Fortificación con fibra dietética como estrategia para aumentar la saciedad: ensayo aleatorizado doble ciego controlado

\section{RESUMEN}

Introducción: Se ha descrito que las fibras dietéticas (FD) podrían tener efectos favorables en el control de sentimientos subjetivos relacionados a sensaciones postprandiales de apetito y saciedad, propiedad que en el contexto epidemiológico actual podrían ser beneficiosos. Objetivo: evaluar el efecto agudo de 3 tipos de FD sobre la percepción subjetiva de sensaciones postprandiales.

Material y Métodos: Estudio randomizado, doble ciego, controlado, cruzado. 21 sujetos sanos con índice de masa corporal entre $18,5-24,9 \mathrm{~kg} / \mathrm{m}^{2}$, fueron asignados aleatoriamente para consumir 4 desayunos separados por a lo menos 2 días. Los desayunos fueron fortificados con $3 \mathrm{~g}$ de: $\beta$-glucano de avena (BETA), pectinas de manzana (PECT), celulosa microcristalina (CELL) y maltodextrina como control (MALT). Los sentimientos subjetivos postprandiales se determinaron con una Encuesta Visual Análoga (EVA) antes del desayuno y a los 15, 30, 60, 90 y 120 minutos.

Resultados: En análisis de varianzas (ANOVA) mostró una diferencia significativa a los 15 minutos $(p<0,05)$ en la percepción de saciedad subjetiva de los desayunos fortificados con pectina de manzana versus $\beta$-glucano de avena. $\beta$-glucano de avena produjo el menor efecto en la saciedad subjetiva $(p<0,05)$. Un ANOVA de medidas repetidas no mostró efectos de los tipos de fibras adicionadas a los desayunos en el sentimiento subjetivo de apetito.

Conclusiones: Las pectinas de manzana parecen ser más efectivas que otros tipos de FD para aumentar la percepción subjetiva de saciedad y disminuir el apetito, especialmente en el periodo postprandial temprano. 


\section{KEYWORDS}

Nutritional

Physiological

Phenomena;

Appetite Regulation;

Appetite;

Satiation;

Satiety Response;

Visual Analog Scale;

Dietary Fiber;

Breakfast;

Adult.

Fortification with dietary fiber as a strategy to increase satiety: controlled double blind randomized trial

\section{A B S TRACT}

Introduction: It has been described that dietary fibers (DF) could have favorable effects in the control of subjective feelings related to appetite/satiety, property that in the current epidemiological context could be beneficial. Objective: to evaluate the acute effect of 3 types of DF on the subjective perception of feelings related to appetite/satiety.

Material and Methods: Randomized, double-blinded, controlled, crossover study. 21 healthy subjects with body mass index between $18.5-24.9 \mathrm{~kg} / \mathrm{m}^{2}$ were randozomized to consume 4 separate breakfasts for at least 2 days. The breakfasts were fortified with $3 \mathrm{~g}$ of: oat $\beta$-glucan (BETA), apple pectins (PECT), microcrystalline cellulose (CELL) and maltodextrin as control (MALT). The subjective feelings of appetite/satiety were determined with a Visual Analog Scale (VAS) before breakfast and at 15, 30, 60, 90 and 120 minutes.

Results: In analysis of variances (ANOVA) showed a significant difference at 15 minutes $(p<0.05)$ in the perception of satiety of breakfast fortified with apple pectin versus oat $\beta$-glucan. Oat $\beta$-glucan produced the least effect on subjective satiety $(p<0.05)$. An ANOVA of repeated measures did not show effects of the types of fibers added to breakfast on the subjective feeling of appetite.

Conclusions: Apple pectins appear to be more effective than other types of DF to increase the subjective perception of satiety and decrease appetite, especially in the early postprandial period.

\section{CITA}

Pino Villalón JL, Rojas Muñoz M, Orellana Saez B, Torres Mejías J. Fortificación con fibra dietética como estrategia para aumentar la saciedad: ensayo aleatorizado doble ciego controlado. Rev Esp Nutr Hum Diet. 2020; 24(4): 336-44. doi: 10.14306/renhyd.24.4.1020

\section{INTRODUCCIÓN}

Según la Organización Mundial de la Salud (OMS), a nivel mundial el 39\% de la población presenta sobrepeso y el $13 \%$ obesidad ${ }^{1}$; en Chile la situación es aún más compleja, según la Encuesta Nacional de Salud (2017) el exceso de peso está presente en el $74 \%$ de la población², prevalencia superior incluso a la descrita en Estados Unidos (71\%) o México $(72,5 \%)$ en el contexto de los países adscritos a la Organización para la Cooperación y el Desarrollo Económicos (OCDE) 3 .

Según una encuesta poblacional realizada en Santiago de Chile, el $72,4 \%$ de los encuestados está tratando de bajar o mantener el peso corporal ${ }^{4}$ realizando algún tipo de actividad, pero la alimentación aún sigue siendo un punto crítico, puesto que, según cifras oficiales, sólo el $5 \%$ de la población mantiene una alimentación saludable ${ }^{5}$. En este contexto, la búsqueda de estrategias que contribuyan a minimizar esta situación epidemiológica posee gran relevancia.

La dieta occidental se caracteriza por ser una alimentación alta en carnes rojas, grasas e hidratos de carbono simples, $y$, por otro lado, deficiente en agua y fibra dietética (FD) ${ }^{6,7}$. De hecho la ingesta de FD en dieta chilena en ningún grupo etario sobrepasa los $13 \mathrm{~g} / \mathrm{dí}^{5}{ }^{5}$, consumo similar a países como Estados Unidos (12-18g/día) ${ }^{8}$ y Reino Unido (14g/ día $)^{9}$, pero que en ningún caso cumplen con una recomendación saludable, las cuales van desde un mínimo de 25 o $30 \mathrm{~g} /$ día $^{10,11}$.

En el contexto epidemiológico actual el consumo de fibra dietética resulta beneficioso por sus múltiples beneficios; disminución del perfil lipídico ${ }^{12}$ y presión arterial ${ }^{13}$, modulación de la microbiota intestinal con un aumento de PYY y GLP-1 ${ }^{14}$, mantención de glicemias estables ${ }^{15}$, y una 
disminución de la ingesta relacionada con un aumento de la percepción subjetiva de saciedad ${ }^{16}$. Por lo que su consumo se ha asociado a una disminución del riesgo de enfermedades no transmisibles ${ }^{17}$.

Actualmente existen estudios que determinan la percepción de saciedad en individuos, utilizando sólo un tipo de fibra dietética ${ }^{18,19}$ o con cereales enteros ${ }^{20}$, pero existen pocos estudios que comparen diferentes tipos de fibras aisladas. Por lo cual, se planteó el objetivo general de evaluar el efecto del consumo de diversos tipos de fibra dietética sobre la predisposición a la ingesta de alimentos. Para cumplir este propósito se plantearon dos objetivos específicos, uno orientado a identificar las sensaciones relacionadas a la percepción de satisfacción postprandial (temprana y tardía) y un segundo objetivo conducente a describir las sensaciones relacionadas a la necesidad de ingerir alimentos (apetito).

Resultados de este tipo de investigaciones son relevantes a la hora de seleccionar alimentos que aumenten la sensación de saciedad en los sujetos, de tal manera que sea posible realizar prescripciones dietéticas más eficientes en sujetos sometidos a dietas para el tratamiento del exceso de peso.

\section{MATERIAL Y MÉTODOS}

Estudio de diseño experimental, doble ciego, aleatorizado y controlado con un diseño cruzado (crossover), cada uno de los sujetos siendo su propio control.

La muestra se calculó esperando una diferencia promedio en la percepción de apetito de 1 punto en la encuesta, con una confianza de $95 \%$, un poder estadístico de $80 \%$ y pérdida de $10 \%$. Dando como resultado 22 sujetos. Se incluyeron sujetos con edad entre 18 y 30 años, de ambos sexos, con un IMC entre $18,5-24,9 \mathrm{~kg} / \mathrm{m}^{2}$, sin patologías que pudiesen alterar la percepción de saciedad o fisiología digestiva (enfermedad celiaca, gastritis, síndrome de vaciamiento rápido, etc.), se excluyeron además fumadores y embarazadas. Los sujetos fueron reclutados mediante convocatoria pública en la comunidad educativa de la Universidad Santo Tomás, Talca, Chile.

El estudio se realizó en las dependencias de la Universidad Santo Tomás, Talca (Laboratorio de Nutrición). Las muestras de fibras fueron adquiridas de Portland SA (Chile) y Xi'an Yaochang Co., Ltd. (China). El resto de alimentos para la preparación de los desayunos se adquirieron en supermercados de la ciudad.

El desayuno estuvo compuesto por una matriz semilíquida (yogur), una sólida (pan blanco con jamón de pavo) y líquida (Té con endulzante) (Tabla 1). La fibra fue adicionada a la matriz semilíquida al $2 \%(3 \mathrm{~g})$.

La aleatorización de la fortificación de los desayunos con FD se realizó utilizando la plataforma www.randomization.com (Suplemento 1), por lo que la entrega de los diferentes tipos de fibra a los sujetos de estudio tuvo un enmascaramiento de doble ciego, puesto que tanto los sujetos en estudio como los coinvestigadores que aplicaron el protocolo desconocían el tipo de fibra entregado, sólo el investigador principal tenía conocimiento de esta información. Las fibras fueron denominadas con letras: $A$, Maltodextrina; $B$, Pectinas de Manzana; C, Celulosa Microcristalina y D, $\beta$-glucano de Avena (Figura 1 ).

Al inicio del primer desayuno se determinaron peso y talla siguiendo las técnicas estandarizadas de la Organización

Tabla 1. Aporte calórico-nutricional de los desayunos.

\begin{tabular}{|c|c|c|c|c|c|c|}
\hline Ingrediente & $\begin{array}{c}\text { Cantidad } \\
(\mathrm{g} / \mathrm{mL})\end{array}$ & $\begin{array}{l}\text { Energía } \\
\text { (kcal) }\end{array}$ & $\begin{array}{l}\text { Proteínas } \\
\text { (g) }\end{array}$ & $\begin{array}{l}\text { Lípidos } \\
\text { (g) }\end{array}$ & $\begin{array}{c}\text { Carbohidratos } \\
\text { (g) }\end{array}$ & $\begin{array}{c}\text { Fibra } \\
\text { (g) }\end{array}$ \\
\hline Pan Molde & 55 & 145 & 4,7 & 1,7 & 27,2 & 2 \\
\hline Jamón de Pavo & 30 & 38 & 5,7 & 1,5 & 0,1 & 0 \\
\hline Yogur + FD 2\% & 150 & 152 & 6,6 & 4 & 22,2 & 3* \\
\hline Té & 200 & - & - & - & - & - \\
\hline & \multirow{2}{*}{ Total } & 335 & 17 & 7,2 & 49,5 & 5 \\
\hline & & & $20 \%$ & $20 \%$ & $60 \%$ & \\
\hline
\end{tabular}

FD: Fibra Dietética; *según aleatorización: Pectinas de Manzana, Celulosa Microcristalina, $\beta$-glucano de avena y maltodextrina (control). 
Figura 1. Diagrama de flujo Protocolo de Estudio.

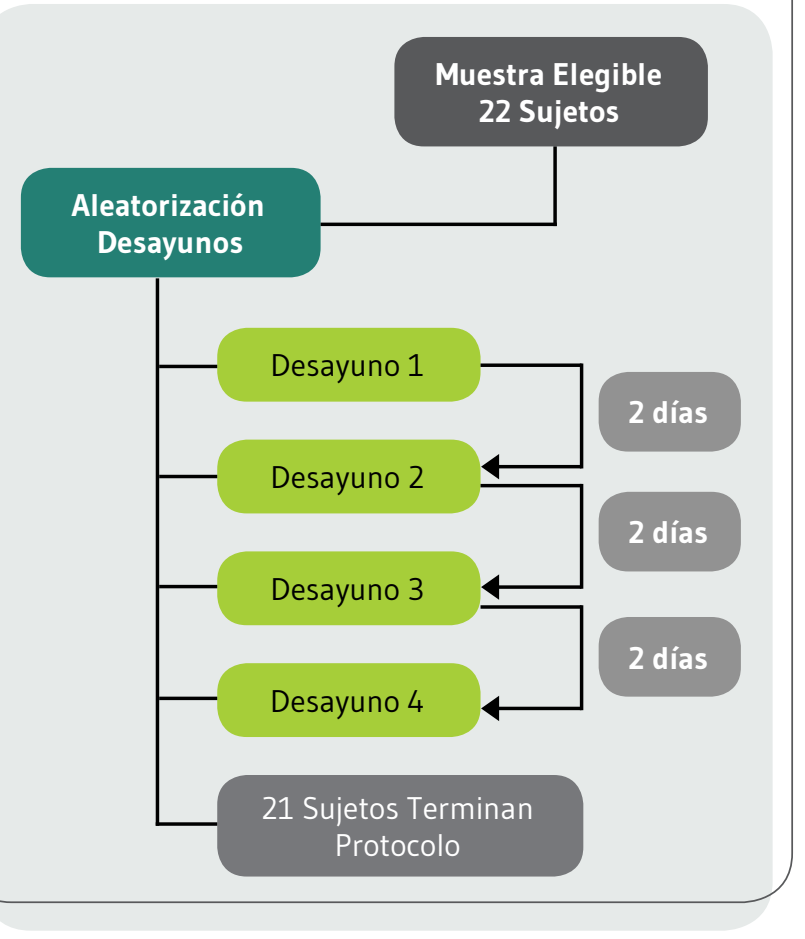

Mundial de la Salud (OMS) ${ }^{21}$. Posteriormente se aplicó una Encuesta Visual Análoga (EVA) (Figura 2) antes del desayuno y a los 15, 30, 60, 90 y 120 minutos luego de la ingestión, procedimiento que se repitió para cada desayuno. Los desayunos de cada sujeto fueron consumidos entre las 8:00 y 9:00 horas, con un tiempo de 10 minutos para la ingesta y cada uno separado por al menos 48 horas.

Se utilizó una EVA ${ }^{22}$ para determinar la percepción de sensaciones postprandiales, este instrumento consulta por las sensaciones subjetivas de hambre, deseo de comer, saciedad y consumo futuro de alimentos. Cada ítem consistió en líneas de $100 \mathrm{~mm}$ con declaraciones opuestas de cada variable (Figura 2). Cada una de las sensaciones fue analizada de manera global (0-120 minutos), como satisfacción postprandial temprana (0-30 minutos) y satisfacción postprandial tardía (60-120 minutos).

Por otro lado, se determinó el puntaje de apetito por la siguiente ecuación: ((hambre + deseo de alimentos + consumo futuro de alimentos + (10-saciedad)) / 4).

Se calculó el puntaje promedio para cada sentimiento subjetivo, además se decidió determinar el área bajo la curva (ABC) para cada sensación postprandial, ya que es un indicador que refleja la percepción total de las sensaciones subjetivas en función del tiempo, y se analizó mediante ANOVA de una vía con post hoc Bonferroni. Por otro lado se analizó el promedio de puntajes de apetito en cada tiempo de medición para cada desayuno con ANOVA de medidas repetidas.

Figura 2. Escala Percepción subjetiva de apetito/saciedad.

\section{DESEO DE COMER}

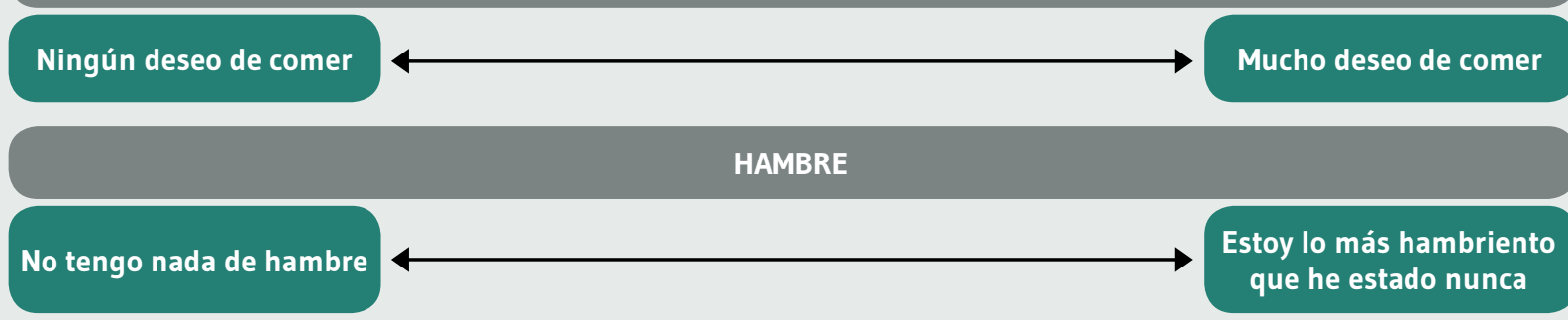

SACIEDAD

No me siento satisfecho

Estoy muy satisfecho

CONSUMO FUTURO DE ALIMENTOS 
Se utilizó 95\% confianza y significación de 0,05. Los datos fueron analizados con el paquete estadístico IBM-SPSS v.19.

El estudio fue aprobado por el comité de ética científico centro sur de la Universidad Santo Tomás N ${ }^{\circ} 110-19$ de fecha 11 de septiembre de 2019. Cada participante fue informado del procedimiento y firmó el consentimiento informado respectivo.

\section{$\longrightarrow$ \\ RESULTADOS}

El periodo de recopilación de información tardó 120 días. Se entregaron 84 desayunos, los que fueron consumidos por 21 voluntarios, sólo un sujeto no completó el consumo de los tiempos de alimentación, resultando un 95\% de adhesión. Del total de participantes 13 fueron mujeres (62\%), la edad promedio fue de $24,19 \pm 3,6$ años. El peso promedio fue de $68,6 \pm 11,2 \mathrm{~kg}$ con una diferencia promedio de $9,9 \mathrm{~kg}(p<0,05)$ a favor de los varones. La talla promedio fue de $167 \pm 8,0 \mathrm{~cm}$ con una diferencia promedio de $10 \mathrm{~cm}(p<0,01)$ también a favor de los varones y el IMC fue de $24,4 \pm 3,7 \mathrm{~kg} / \mathrm{m}^{2}$ sin diferencias por sexo. La ocupación de todos los participantes fue estudiante de educación superior.

Según los puntajes de la EVA se logró apreciar una diferencia significativa en la sensación subjetiva de saciedad de los voluntarios en el periodo postprandial temprano, específicamente a los 15 minutos luego del consumo del desayuno. El desayuno fortificado con $\beta$-glucano de avena produjo una sensación de saciedad significativamente inferior al desayuno con incorporación de pectinas de manzana $(p=0,007)$ y maltodextrina $(p=0,008)$ (Figura 3$)$.

No se encontró diferencias en deseo de comer, hambre, saciedad o consumo futuro de alimentos en el $A B C$ durante el periodo global (0-120 minutos). Al realizar el análisis en faces separadas, postprandial temprano (0-30 minutos) y tardío (60-120 minutos), para los diferentes sentimientos subjetivos, se logró observar una diferencia en el periodo de postprandial temprano $(p<0,05)$. El análisis post hoc identificó diferencias significativas entre los desayunos con inclusión de $\beta$-glucano de avena y con adición de pectinas de manzana (Tabla 2 y Tabla 3), generando este último mayor saciedad a corto plazo $(p<0,05)$.

El puntaje de apetito se determinó antes del desayuno y en 5 tiempos; 15, 30, 60, 90 y 120 minutos. Los puntajes promedios de apetito para cada tipo de fibra dietética y maltodextrina no se diferenciaron en ningún tiempo de medición (Tabla 4).

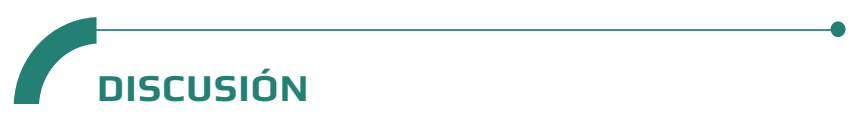

El presente estudio evaluó la percepción subjetiva de sensaciones postprandiales de diferentes tipos de fibras dietéticas adicionadas a desayunos de igual estructura y aporte calórico nutricional. Los resultados sugieren que los sentimientos subjetivos no difieren mucho entre los distintos tipos de FD adicionada. Sólo la saciedad postprandial temprana, es decir, durante el periodo menor a 30 minutos luego de la ingesta, cambió por efecto de las FD. Esta observación sugiere que el tipo de FD adicionada al desayuno no produce una diferencia significativa en la percepción de sentimientos subjetivos postprandiales.

La determinación de la percepción subjetiva de sensaciones postprandiales se ha protocolizado con anterioridad generando guías para su determinación ${ }^{23}$, dichas recomendaciones fueron de soporte para la realización de esta investigación.

La percepción subjetiva de sensaciones postprandiales producida por un tiempo de alimentación podría ser diferente cuando se determina en condiciones controladas de laboratorio o en el hogar, pero estudios previos han descrito que el lugar de consumo no influiría en la percepción de apetito/ saciedad ${ }^{20}$, por lo que, los resultados obtenidos podrían ser extrapolados independiente del lugar de consumo.

Nuestro estudio destacó un efecto relevante de la incorporación de pectinas de manzana al desayuno, generando menor percepción subjetiva de hambre, deseo de comer y apetito, y una mayor sensación subjetiva de saciedad especialmente en el periodo de postprandial temprano (15 minutos). Las pectinas, fibras dietéticas solubles y generadoras de viscosidad, disminuyen la saciedad en mayor medida durante la primera hora luego de la ingestión en comparación con otros tipos de FD, logrando una disminución de la percepción subjetiva de apetito entorno al $17 \%{ }^{16}$; resultado descrito en otros estudios ${ }^{24}$, incluso generando mayor saciedad que alimentos proteicos ${ }^{25}$, pero su influencia en la ingesta energética es controversial ${ }^{26}$.

Un resultado interesante fue la baja sensación de saciedad que produjo el $\beta$-glucano de avena, se ha descrito anteriormente que este tipo de fibra incorporada en los alimentos podría reducir puntajes de EVA en hambre y consumo futuro de alimentos, y por otro lado producir un aumento de saciedad subjetiva, incluso disminuyendo la ingesta energética ${ }^{27}$, pero en este estudio no se logró comprobar estos resultados, tal vez, se podría atribuir a la matriz en la cual se adicionó la FD, el $\beta$-glucano tiene una mejor efectividad 
Figura 3. Puntaje de sensaciones postprandiales según tipo de fibra dietética adicionada al desayuno.
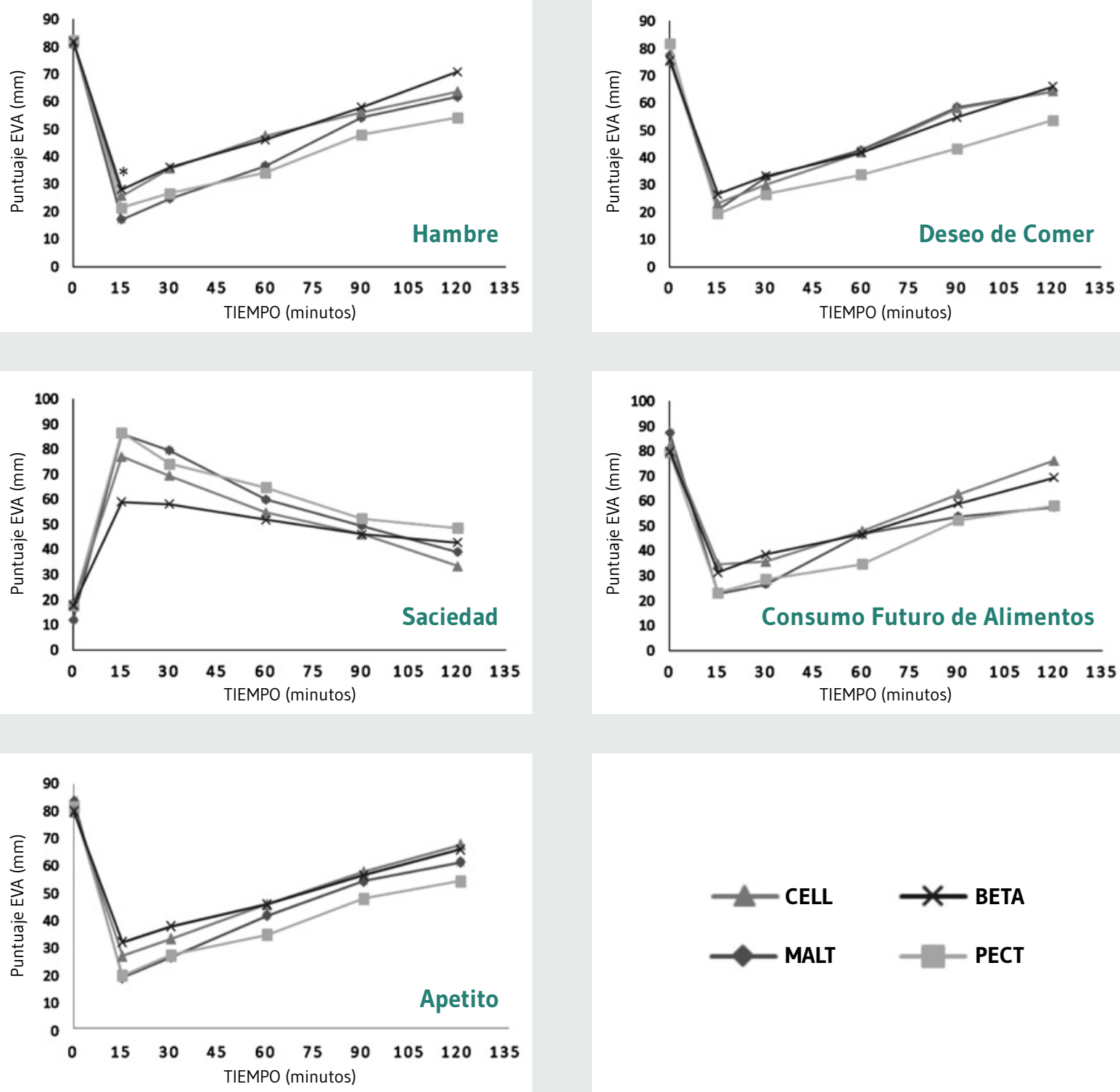

CELL: Celulosa microcristalina; MALT: Maltodextrina; BETA: Beta-glucano de avena; PECT: Pectinas de manzana.

en la producción de saciedad subjetiva cuando se adiciona a matrices líquidas 28 .

Los efectos de las FD sobre la percepción subjetiva de sensaciones postprandiales podrían contribuir a la disminución de la ingesta, como se ha determinado en otros estu$\operatorname{dios}^{29}$ incluso en niños ${ }^{30}$, efecto que se podría traducir en una disminución del peso corporal ${ }^{31}$; pero esta reducción dependería de múltiples factores relacionados al tipo de fibra, cantidad entregada, tratamiento hipocalórico paralelo, entre otros ${ }^{32}$. Pero de todas formas, la ingesta de FD contribuye a la reducción del riesgo de enfermedades no transmisibles ${ }^{33}$, por lo que se debe promover su ingesta. 
Tabla 2. Área bajo la curva de cada sensación subjetiva según tipo de fibra dietética adicionada al desayuno durante el periodo postprandial temprano (0-30 minutos).

\begin{tabular}{l|c|c|c|c|c} 
& Maltodextrina & Pectinas & Celulosa & $\beta$-glucano & p-valor \\
\hline Deseo de comer & $115 \pm 94,5$ & $103 \pm 84,1$ & $130 \pm 76,3$ & $139 \pm 107$ & 0,746 \\
\hline Hambre & $115 \pm 97,1$ & $99 \pm 80,6$ & $112 \pm 63,1$ & $127 \pm 86,5$ & 0,593 \\
\hline Saciedad & $381 \pm 71,9^{\mathrm{a}}$ & $368 \pm 97,6^{\mathrm{b}}$ & $336 \pm 122$ & $279 \pm 140^{\text {ab }}$ & $\mathbf{0 , 0 1 8}$ \\
\hline Consumo futuro de alimentos & $105 \pm 83,6$ & $113 \pm 101$ & $151 \pm 98,8$ & $150 \pm 97$ & 0,255
\end{tabular}

Promedio \pm Desviación Estándar; ANOVA de una vía - post hoc Bonferroni. Letras superíndices muestran parejas con diferencias significativas $p<0,05$.

Tabla 3. Área bajo la curva de cada sensación subjetiva según tipo de fibra dietética adicionada al desayuno durante el periodo postprandial tardío (60 -120 minutos).

\begin{tabular}{l|c|c|c|c|c|} 
& Maltodextrina & Pectinas & Celulosa & $\beta$-glucano & p-valor \\
\hline Deseo de comer & $265 \pm 145$ & $211 \pm 147$ & $263 \pm 121$ & $263 \pm 136$ & 0,525 \\
\hline Hambre & $258 \pm 142$ & $220 \pm 158$ & $264 \pm 119$ & $281 \pm 131$ & 0,536 \\
\hline Saciedad & $207 \pm 133$ & $236 \pm 154$ & $185 \pm 103$ & $205 \pm 117$ & 0,638 \\
\hline Consumo futuro de alimentos & $245 \pm 132$ & $235 \pm 154$ & $302 \pm 124$ & $280 \pm 139$ & 0,376 \\
\hline
\end{tabular}

Promedio \pm Desviación Estándar; ANOVA de una vía.

Tabla 4. Puntaje promedio de apetito en cada tiempo de medición según tipo de fibra adicionada al desayuno.

Tiempo desde ingesta del desayuno

\begin{tabular}{|c|c|c|c|c|c|c|}
\hline \multicolumn{2}{|c|}{ Desayuno } & $15 \mathrm{~min}$ & $30 \mathrm{~min}$ & $60 \mathrm{~min}$ & $90 \mathrm{~min}$ & $120 \mathrm{~min}$ \\
\hline \multicolumn{2}{|c|}{ Maltodextrina } & $2,25 \pm 2,19$ & $2,86 \pm 2,37$ & $4,31 \pm 2,97$ & $5,49 \pm 3,24$ & $6,05 \pm 2,86$ \\
\hline \multicolumn{2}{|c|}{ Pectinas Manzana } & $2,05 \pm 1,85$ & $2,79 \pm 2,29$ & $3,45 \pm 2,66$ & $4,69 \pm 3,27$ & $5,33 \pm 3,54$ \\
\hline \multicolumn{2}{|c|}{ Celulosa Microcristalina } & $2,67 \pm 2,05$ & $3,3 \pm 2,01$ & $4,57 \pm 2,32$ & $5,77 \pm 2,58$ & $6,77 \pm 2,68$ \\
\hline \multicolumn{2}{|c|}{ Beta-Glucano Avena } & $3,29 \pm 2,39$ & $3,68 \pm 2,24$ & $4,57 \pm 2,31$ & $5,64 \pm 2,76$ & $6,49 \pm 2,9$ \\
\hline \multirow{3}{*}{ p-valor } & Efecto Fibra & \multicolumn{5}{|c|}{0,152} \\
\hline & Efecto Tiempo & \multicolumn{5}{|c|}{0,000} \\
\hline & Interacción & \multicolumn{5}{|c|}{0,613} \\
\hline
\end{tabular}

Promedio \pm Desviación Estándar; ANOVA Medidas repetidas 2 factores. 
Entre las limitaciones de la investigación podemos mencionar que, si bien un ensayo cruzado tiene validez metodológica, también presenta inconvenientes; por ejemplo, a pesar de la aleatorización, el orden de los desayunos podría afectar el resultado.

\section{$\longrightarrow$ \\ CONCLUSIONES}

Según nuestros hallazgos, las pectinas, fibra dietética soluble, parece ser más efectiva que otros tipos de FD para aumentar la percepción subjetiva de saciedad y disminuir el apetito, especialmente en el periodo postprandial temprano. Futuras investigaciones podrían abordar la incorporación de estas fibras en matrices alimentarias, evaluando la percepción organoléptica del producto, así como su efecto saciante.

\section{CONTRIBUCIÓN DE AUTORÍA}

JLPV fue el responsable de la redacción del proyecto, planificación del ensayo y redacción del manuscrito.

MRM y BOS realizaron el trabajo de campo, preparación de desayunos, aplicación de encuestas.

JTM participó en la redacción y evaluación del manuscrito.

JLPV, MRM, BOS y JTM revisaron y aprobaron el manuscrito final.

\section{FINANCIACIÓN}

Este trabajo recibió apoyo del Centro de Estudios en Alimentación y Nutrición (CENAN), Chile y Escuela de Nutrición y Dietética de la Universidad Santo Tomás, Talca, Chile.

\section{Conflicto de intereses}

Los autores expresan que no existen conflictos de interés al redactar el manuscrito.

\section{REFERENCIAS}

(1) Organización Mundial de la Salud, Obesidad y sobrepeso, 16 de febrero de 2018, disponible en: https://www.who.int/es/ news-room/fact-sheets/detail/obesity-and-overweight

(2) Gobierno de Chile, Ministerio de Salud, Encuesta Nacional de Salud 2016-2017, disponible en: https://www.minsal.cl/ wp-content/uploads/2017/11/ENS-2016-17_PRIMEROSRESULTADOS.pdf

(3) Organisation for Economic Co-operation and Development (OCDE) (2019), The Heavy Burden of Obesity: The Economics of Prevention, OECD Health Policy Studies, OECD Publishing, Paris, disponible en: https://doi.org/10.1787/67450d67-en

(4) Estudio Movimiento es Felicidad, GFK-ADIMARK, disponible en: www.adimark.cl/es/noticias/index.asp?id=118

(5) Gobierno de Chile, Ministerio de Salud, Encuesta Nacional de Consumo Alimentario 2014, disponible en: https://www. minsal.cl/enca/

(6) Ciprián D, Navarrete-Muñoz EM, Garcia de la Hera M, GiménezMonzo D, González-Palacios S, Quiles J, Vioque J. Patrón de dieta mediterráneo y occidental en población adulta de un área mediterránea; un análisis clúster. Nutr Hosp. 2013; 28: 1741-49.

(7) Urquiaga I, Guasch V, Marshall G, San Martín A, Castillo Ó, Rozowski J, Leighton F. Effect of Mediterranean and Occidental Diets, and Red Wine, on Plasma Fatty Acids in Humans: An Intervention Study. Biological Research. 2004; 37(2): 253-61.

(8) King DE, Mainous AG 3rd, Lambourne CA. Trends in dietary fiber intake in the United States, 1999-2008. J Acad Nutr Diet. 2012; 112(5): 642-8.

(9) Bates B., Lennox, A., Prentice, A., Bates, C. J., Page, P., Nicholson, S. \& Swan, G. (Eds.). (2014). National Diet and Nutrition Survey: Results from Years 1-4 (combined) of the Rolling Programme (2008/2009-2011/12). Executive Summary. Public Health England.

(10) U.S. Food and Drug Administration (FDA), Dietary Fiber, disponible en: https://www.accessdata.fda.gov/scripts/ InteractiveNutritionFactsLabel/factsheets/Dietary_Fiber.pdf

(11) Delzenne NM, Olivares M, Neyrinck AM, Beaumont M, Kjølbæk L, Meinert Larsen T, Benítez-Páez A, Romaní-Pérez $M$, GarciaCampayo V, Bosscher D, Sanz Y, van der Kamp JW. Nutritional interest of dietary fiber and prebiotics in obesity: Lessons from the MyNewGut consortium. Clin Nutr. 2020; 39(2): 414-24.

(12) Ho H, Sievenpiper J, Zurbau A, Blanco-Mejia S, Jovanovski E, Au-Yeung $F$, Jenkins Alexandra, Vuksan Vladimir. The effect of oat $\beta$-glucan on LDL-cholesterol, non-HDL-cholesterol and apoB for CVD risk reduction: a systematic review and metaanalysis of randomised-controlled trials. Br ] Nutr. 2016; 116: 1369-82.

(13) Aleixandre A, Miguel M. Dietary fiber and blood pressure control. Food Funct. 2016; 7(4): 1864-71.

(14) Simpson HL, Campbell BJ. Review article: dietary fibremicrobiota interactions. Aliment Pharmacol Ther. 2015; 42(2): 158-79.

(15) Shen XL, Zhao T, Zhou Y, Shi X, Zou Y, Zhao G. Effect of Oat $\beta$-Glucan Intake on Glycaemic Control and Insulin Sensitivity of 
Diabetic Patients: A Meta-Analysis of Randomized Controlled Trials. Nutrients. 2016; 8(1): 39.

(16) Wanders AJ, van den Borne JJCG, de Graaf C, Hulshof T, Jonathan $M$, et al. Effects of dietary fibre on subjective appetite, energy intake and body weight: a systematic review of randomized controlled trials. Obes Rev. 2011; 12(9): 724-39.

(17) Wendy J. Dahl, Maria L. Stewart, Position of the Academy of Nutrition and Dietetics: Health Implications of Dietary Fiber. J Acad Nutr Diet. 2015; 115(11): 1861-1870.

(18) Aoe S, Ikenaga T, Noguchi H, Kohashi C, Kakumoto K, Kohda $N$. Effect of cooked white rice with high $\beta$-glucan barley on appetite and energy intake in healthy Japanese subjects: a randomized controlled trial. Plant Foods Hum Nutr. 2014; 69(4): 325-30.

(19) Hume MP, Nicolucci AC, Reimer RA. Prebiotic supplementation improves appetite control in children with overweight and obesity: a randomized controlled trial. Am J Clin Nutr. 2017; 105(4): 790-9.

(20) Pasman WJ, Hendriks HFJ, Minekus MM, de Ligt RAF, ScholtesTimmerman MJ, Clabbers NDS, Leonards NM, Johnson J, Bellmann S. Subjective feelings of appetite of wholegrain breakfasts evaluated under controlled, laboratory and 'at home' conditions. Physiol Behav. 2018; 194: 285-91.

(21) WHO STEPS Guide to Physical Measurements, Training \& Practical Guides, disponible en: https://www.who.int/ ncds/surveillance/steps/Section $\% 204 \% 20$ Step $\% 202 \% 20$ Physical\%20Measurements.pdf

(22) Ibarra A, Astbury NM, Olli K, Alhoniemi E, Tiihonen K. Effect of Polydextrose on Subjective Feelings of Appetite during the Satiation and Satiety Periods: A Systematic Review and MetaAnalysis. Nutrients. 2016; 8(1): 45.

(23) Gibbons C, Finlayson G, Dalton M, Caudwell P, Blundell JE. Metabolic Phenotyping Guidelines: Studying eating behaviour in humans. J Endocrinol. 2014; 222(2): G1-12

(24) Wanders AJ, Feskens EJ, Jonathan MC, Schols HA, de Graaf C, Mars M. Pectin is not pectin: a randomized trial on the effect of different physicochemical properties of dietary fiber on appetite and energy intake. Physiol Behav. 2014; 128: 212-9.
(25) Adam CL, Gratz SW, Peinado DI, Thomson LM, Garden KE, Williams PA, Richardson AJ, Ross AW. Effects of Dietary Fibre (Pectin) and/or Increased Protein (Casein or Pea) on Satiety, Body Weight, Adiposity and Caecal Fermentation in High Fat Diet-Induced Obese Rats. PLoS One. 2016; 11(5): e0155871.

(26) Hopkins M, Beaulieu K, Myers A, Gibbons C, Blundell JE. Mechanisms responsible for homeostatic appetite control: theoretical advances and practical implications. Expert Rev Endocrinol Metab. 2017; 12(6); 401-15.

(27) Aoe S, Ikenaga T, Noguchi H, Kohashi C, Kakumoto K, Kohda $N$. Effect of cooked white rice with high $\beta$-glucan barley on appetite and energy intake in healthy Japanese subjects: a randomized controlled trial. Plant Foods Hum Nutr. 2014; 69(4): 325-30.

(28) Pentikäinen S, Karhunen L, Flander L, Katina K, Meynier A, Aymard P, Poutanen K. Enrichment of biscuits and juice with oat $\beta$-glucan enhances postprandial satiety. Appetite. 2014; 75: 150-6.

(29) Barone Lumaga R, Azzali D, Fogliano V, Scalfi L, Vitaglione P. Sugar and dietary fibre composition influence, by different hormonal response, the satiating capacity of a fruit-based and a $\beta$-glucan-enriched beverage. Food Funct. 2012; 3(1): 67-75.

(30) Hume MP, Nicolucci AC, Reimer RA. Prebiotic supplementation improves appetite control in children with overweight and obesity: a randomized controlled trial. Am J Clin Nutr. 2017; 105(4): 790-9.

(31) Ho IH, Matia-Merino L, Huffman LM. Use of viscous fibres in beverages for appetite control: a review of studies. Int ] Food Sci Nutr. 2015; 66(5): 479-90.

(32) Namazi N, Larijani B, Azadbakht L. Are Isolated and Complex Fiber Supplements Good Choices for Weight Management? A Systematic Review. Arch Iran Med. 2017; 20(11): 704-13.

(33) Silva FM, Kramer CK, de Almeida JC, Steemburgo T, Gross $\mathrm{JL}$, Azevedo MJ. Fiber intake and glycemic control in patients with type 2 diabetes mellitus: a systematic review with metaanalysis of randomized controlled trials. Nutr Rev. 2013; 71(12): 790-801. 